\title{
Algunas disertaciones sobre la enseñanza de las ciencias desde la Educación Ambiental, una propuesta para Educación no Formal
}

\author{
Some dissertations about the teaching from the environmental education, an approach \\ to Informal Education
}

Yisaidy Chaparro Díaz, María Suárez Chaparro y Quira Alejandra Sanabria

Universidad Pedagógica Nacional. yisaidy@gmail.com, msuarez470@gmail.com y qsanabria@gmail.com

\section{Resumen}

El presente trabajo presenta algunas disertaciones sobre la enseñanza de las ciencias desde la educación ambiental en contextos no escolarizados, a partir del uso de una red que aborda conceptos estructurantes desde Química, Física y Biología, visto en un contexto social, económico y político determinado, donde se establece cómo interactúa en el sistema tierra. La característica fundamental es la formación de sujetos que frente a la devastación de la naturaleza promueven su respeto e integridad, mediante la comprensión de las problemáticas ambientales y la participación en la toma de decisiones para su resolución.

\section{Palabras clave}

Enseñanza de las ciencias, Educación ambiental y Educación no formal.

\section{Abstract}

This paper presents some dissertations about the teaching from the environmental education on informal contexts, induding the use of a conceptual network that get down structural concepts from Chemistry, Physics and Biology, from a social, economic and political context, which stets how the earth system interacts. The fundamental characteristic is the formation of subjects compared to the devastation of nature promotes respect and integrity, through understanding of environmental issues and participation in making decisions for resolution.

\section{Keywords}

Science education, environmental education, and informal education.

\section{Introducción}

A lo largo del siglo XX la enseñanza de las ciencias estaba vinculada únicamente a la formación en "artes mecánicas", ciencias militares o agricultura. Posteriormente surge la necesidad de nuevas profesiones con formación cientíica, hacia el año de 1860 en Alemania, se realiza una reestructuración a los programas en el área cientúfica con la separación de las tres disciplinas Física, Química y Biología, la cual se ha mantenido sin cambios relevantes hasta ahora (Sanmartí, 2002). Sin embargo la demanda social no cesó, antes bien se globalizó y a partir de ello se comienza a investigar los problemas relacionados con la enseñanza de las ciencias, para el planteamiento de nuevas propuestas curriculares. 
De modo que se replantea el objeto de la enseñanza de las ciencias, ya no es solamente preparar a los que en el futuro serán estudiantes universitarios de ciencias, sino a todos los ciudadanos en general, lo que obliga a revisar de manera precisa los planteamientos tradicionales y donde se hace necesario un compromiso de ésta en campos como la educación ambiental, el desarrollo de la salud, la formación hacia la paz y el civismo (Sanmartí, 2002). Los propios contextos se constituyen así en ámbitos de aprendizaje en los que confluyen todos los aspectos del proceso educativo, desde su formulación (diagnóstico de los problemas), hasta su fase final (propuestas de solución y toma de decisiones) (Novo, 2005).

A pesar de las diversas innovaciones e investigaciones en el campo de la didáctica, se encuentra que aun los resultados en las aulas de clase siguen siendo muy distantes de lo que se espera, identificándose desconexión entre la realidad, el entomo educativo, los conocimientos científicos y la educación ambiental. En esta medida es importante resaltar el papel que la Educación Ambiental (en adelante EA) ha desempeñado como estructura de mediación entre los procesos de conocimiento que se dan en la escuela, el medio ambiente real y las experiencias practicas que tienen lugar en el ámbito ciudadano o en centros educativos extraescolares, permitiendo las reflexiones críticas y convirtiéndose en un referente del cambio respecto el imaginario colectivo del papel de la educación, su alcance y la necesaria integración de los procesos educativos en su contexto real (Novo, 2005)

El aprendizaje que de aquí se deriva implica, por tanto, la adquisioión de mucho más que unos meros conocimientos sobre naturaleza o sociedad: se trata de un meta-aprendizaje acerca de las posibilidades de comprender el mundo y el papel de los ciudadanos en él mediante la participación, la práctica activa, la resolución de problemas y la toma de decisiones (Novo, 2005). Frente a esta postura surgen los siguientes auestionamientos; ¿De qué forma se permite a la población la construcción de herramientas para afrontar y ayudar a la compresión de los problemas que hoy son objeto de discusión? (Sanmartí, 2002), y espećficamente, ¿qué importancia tiene la enseñanza de la educación ambiental más allá de la educación formal?

\section{Algunas tendencias de la Educación Ambiental (EA)}

La educación ambiental (EA) es reconocida como una dimensión esencial de la educación global por el papel dave que desempeña en la construcción de nuevas actitudes, comportamientos y valores tanto del individuo como de las comunidades con el medio socio ambiental (Novo, 2005 y Sauvé, 1999). Sin embargo, a través de su historia, este papel ha sido abordado de modo diferente: como contenido, como proceso, como orientación curricular, como una materia. (Sauvé, 1999).

Si bien la educación ambiental ha sido caracterizada por una visión instrumentalista, centrada principalmente en la resolución de problemas, para lograr un uso más racional de los recursos naturales y su protección, hoy día ha de constituirse en un verdadero motor de transformaciones, por su finalidad y por los procesos que desencadena, se presenta como un importante factor de cambios: en el medio ambiente, al contribuir a mejorar su estado de deterioro, al proteger la biodiversidad, respetándolo, preservándolo y reconociendo el medio desde su multiplicidad, en la población, al estimular el desarrollo cognitivo, afectivo, social y moral, a modo de forjar nuevas relaciones con el medio de vida (Sauvé, 1999).

\section{La Educación Ambiental no formal como eje de cambio social}

Considerando la educación ambiental como un requisito indispensable para superar la crisis ambiental y para la construcción de nuevos modelos de desarrollo, se ha realizado 
significativamente una mayor labor de educación ambiental formal, en Colombia abordada en su mayoría con la implementación de PRAES (Proyectos Ambientales Escolares) en las instituciones educativas, en este sentido, la población escolar sigue siendo el sector al que van dirigidas la mayoría de las iniciativas en educación ambiental, lo que limita la capacidad de acción, por un lado al exduir al resto de la sociedad, y por otro por la escasa incidencia de los niños sobre las decisiones y comportamientos de impacto en el ambiente.

De igual manera, los saberes de la EA enseñados tradicionalmente en el ámbito formal requieren ser revisados a fondo, no tiene sentido abordar la EA con la misma estructura disciplinar que a finales del siglo XIX, cuando el numero de conocimientos científicos ha aumentado exponencialmente y en el caso de las ciencias ambientales se exige un carácter interdisciplinar (Sanmartí, 2002).

En efecto, si la EA formal se ve limitada por las restricciones que impone la organización institucional y es, por tanto, esencialmente informativa y formativa, y se basa en la adquisición de conocimientos, la EA no formal resulta especialmente eficaz gracias al modo en que utiliza los mecanismos experienciales de aprendizaje y al énfasis que pone en que quienes aprenden desarrollen una "conciencia participativa"(Novo, 2005)

Con respecto a los aprendizajes, de los problemas y tópicos ambientales, como centros de interés hace que, en este tipo de educación, no haya asignaturas y desaparezcan las divisiones disciplinarias propias de la educación formal, favoreciendo procesos de interpretación transdiciplinar. Lo que permite abordar las cuestiones ambientales en toda su complejidad y más cuando a lo largo de estas décadas, no han sido muchas las posibilidades potenciales de la educación ambiental no formal que se han ido materializando, aspecto que ha convertido a este movimiento en uno de los ejes de cambio y transformación social más importantes del momento actual.

\section{La dimensión socio ambiental}

A principios de la década de los setenta surgió en los ambientes científicos de los países industrializados la preocupación ante el hecho de la crisis ambiental que se detectaba en el planeta. No obstante, la conciencia del problema ambiental y las acciones llevadas a cabo en los últimos años, no han podido revertir las fuertes tendencias de degradación, por lo que las consecuencias de este hecho han conducido a aspirar en la "necesidad de un conjunto de fuerzas colectivas que atenúen, comijan y transformen cuanto antes esta incidencia negativa sobre el equilibrio ecológico y la equidad social" (Novo, 2005).

De esta manera, se apunta a desarrollar sociedades responsables, ayyo despliegue significa, reconstruir en una armonía la compleja red de relaciones e interacciones que existen entre las personas, la sociedad y los sistemas ecológicos. Este hecho implica contemplar además el entomo como un factor que a lo largo de la historia ha contribuido a formar los modos de vida y las relaciones entre los humanos, como analizar los cambios producidos por éstos en su entomo y las consecuencias que han tenido dichos cambios.

En este sentido, la EA es una estrategia que permite interrelacionar las fuerzas de cambio, tomando como referente el pensamiento y el análisis de los problemas a escala planetaria pero desarrollando, al mismo tiempo, propuestas de acción contextualizadas que contemplen las necesidades, potencialidades y características de cada entomo, haciendo manifiesto una nueva mirada en el protagonismo creciente de una sociedad civil democrática (Novo, 2005). 
Atendiendo al carácter sistémico del ambiente, la educación no formal cobra especial valor, al estar referida a todas aquellas iniciativas dirigidas a facilitar a los niños, jóvenes y adultos no escolarizados o fuera de actividades de la Educación Formal, acciones de carácter ambiental, que van encaminadas a la búsqueda de niveles apropiados a la conservación, mejoramiento y defensa del ambiente.

\section{Una propuesta para la formación no formal en la Educación Ambiental}

El considerar la educación para la población en general significa pensar en espacios no formales puesto que muchas personas no hacen parte del sistema educativo formal, pero no por ello deben ser exduidos de una necesidad dedarada para el mejoramiento de la calidad de vida de la población del planeta, en esta perspectiva se presenta esta propuesta de intervención para comunidades.

Es evidente que no se puede enseñar todo en la escuela, y por tanto un problema que plantea la didáctica de las ciencias, es definir los criterios de los conceptos estructurantes para la enseñanza de las ciencias (Sanmartí, 2002), de este modo, haciendo uso de un enfoque holístico, se propone abordar la educación ambiental desde la globalidad sin entrar en detalles, como lo expresaría Novo desde lo "glocal", pero permitiendo a la población la construcción de herramientas para afrontar y ayudar a la compresión de las problemáticas ambientales.

En esta medida, es importante tomar como punto de partida, cómo la necesidad de una población requiere de especialistas que sean capaces de contextualizar el trabajo conceptual, para ello, se recurre a la elaboración de una red conceptual, aryos conceptos estructurantes muestren interacción en la dinámica del sistema tienra y confluyan en un contexto social, político y económico propio.

La figura 1 presenta los conceptos estructurantes que se proponen para la enseñanza de las ciencias a través de la educación ambiental como propuesta para la educación no formal, alrededor de los cuales se pueden construir y generar los demás, ante la interacción en el sistema tiema conformada por la antroposfera, hidrosfera, atmosfera, litosfera y biosfera, donde la interacción entre la antroposfera y las demás esferas generalmente se asume como la interacción sociedad naturaleza, utilizando la ciencia y la tecnología. 


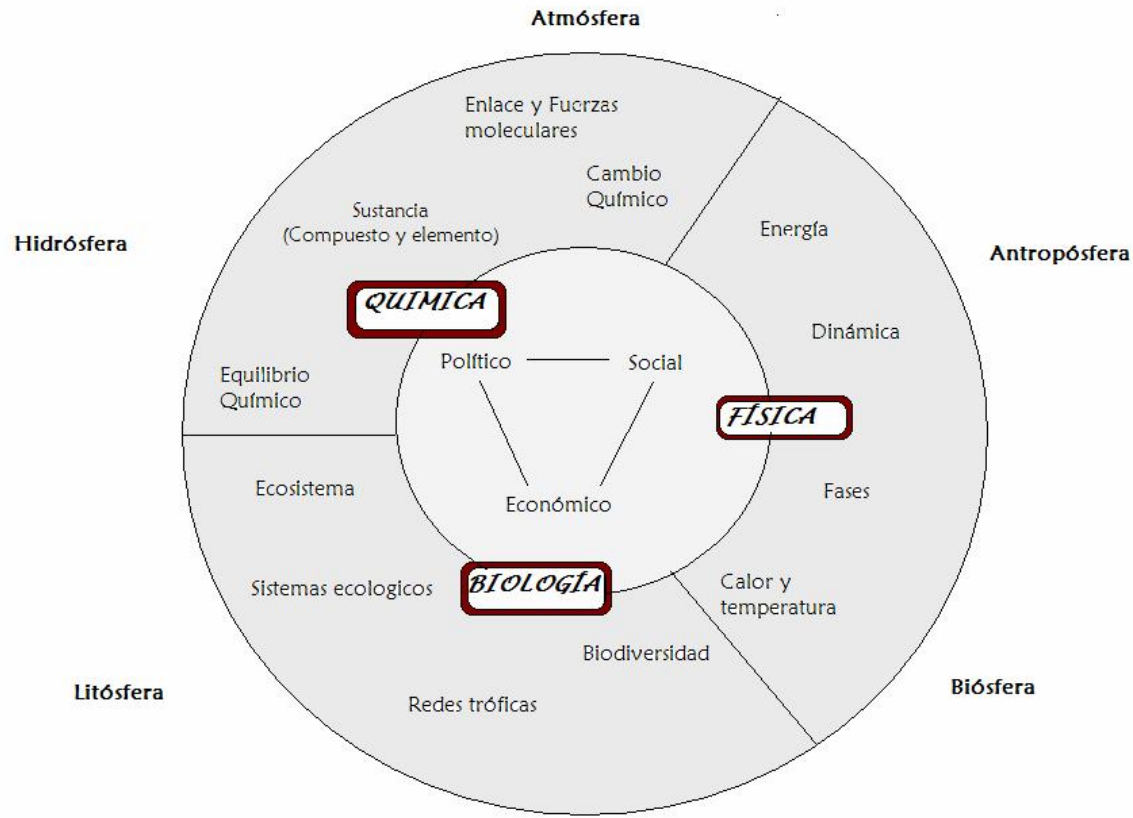

Figura 1. Conceptos estructurantes para la enseñanza de la Educación Ambiental No Formal.

El concepto de energía como concepto estructurante desde la física permite explicar los fenómenos ocurridos a nivel atmosférico (radiación solar, presión atmosférica, evaporación, humedad atmosférica) su alteración causa problemas en la capa de ozono, relacionados también con los conceptos de calor y temperatura, que a su vez pueden alterar el equilibrio dinámico (viento, nubosidad, precipitación).

Se aborda los siguientes conceptos estructurantes como programa de investigación cient́fica por considerarse que entre ellos hay un fortalecimiento del querpo teórico más que una sustitución de los mismos (Lakatos, 1983): Sustancia, desde la química, como objeto de conocimiento e investigación propuesto por los especialistas desde las interacciones: sustancia-sustancia y sustancia-energía y el cambio químico que considera los intercambios de energía entre sistema y ambiente.

La biología estructura el estudio de los organismos vivos y las interrelaciones entre ellos mismos con el ambiente físico y biológico a su alrededor; el ecosistema como unidad de estudio de los sistemas ecológicos y su estructura trófica (cadenas y redes tróficas). La biodiversidad como característica biológica que hace referencia al grado de heterogeneidad existente en los distintos niveles de organización que presentan los seres vivos.

El centro de la esfera constituye el contexto, no es posible definir las finalidades de la EA sin tener en cuenta las realidades económicas, sociales y políticas de cada sociedad y los objetivos que ésta se haya fijado para su desarrollo (Novo, 2005). De este modo, tanto los ecosistemas como las implicaciones sociales, políticas y económicas, se encuentran en constante interacción afectándose mutuamente, en donde el equilibrio de la relación condiciona la capacidad de soporte ambiental.

\section{Consideraciones finales}

- La EA ha de alcanzar a todos los sectores de la población, especialmente aquellos en quienes recae la toma de decisiones y tienen mayor responsabilidad, en tanto que es necesario el uso de algunos conceptos estructurantes en las ciencias que induyen las disciplinas como química, física y biología para intervenir en la resolución de los problemas. 
- Los sistemas educativos formales y no formales comparten con los procesos de enseñanza de las ciencias la responsabilidad de hacer extensión social y vincular a toda la población. Las posibilidades de interacción social y de participación que se generan en la comunidad suponen un gran potencial para el desarrollo de una EA, aquella que se da aun auando no hay una planificación educativa y si se considera que ha de ser un proceso de aprendizaje continuo que puede darse en contextos diversos y que sus estrategias han de ser múltiples.

\section{Bibliografía}

Lakatos, I. (1983). La metodología de los Programas de Investigación Científica. Madrid, Alianza Regional.

Novo Villaverde, M (2005). Educación ambiental formal y no formal: dos sistemas complementarios. Revista de educación, (338), 145-165.

Sanmartí, N. (2002). Enseñar ciencias en los inicios del siglo XXI. Didáctica de las ciencias en educación secundaria obligatoria. (pp. 11-29). Madrid.

Sauvé, L. (1999). La educación ambiental entre la modemidad y la pos modernidad: en busca de un marco de referencia educativo integrador, Tópicos en Educación Ambiental. (1) 2, 9-35. 